\section{Hypofunction in the posterior cingulate gyrus correlates with disorientation for time and place in Alzheimer's disease}

\author{
Nobutsugu Hirono, Etsuro Mori, Kazunari Ishii, Yoshitaka Ikejiri, Toru Imamura, \\ Tatsuo Shimomura, Mamoru Hashimoto, Hikari Yamashita, Masahiro Sasaki
}

\begin{abstract}
The relation between orientation for time and place and regional cerebral glucose metabolism was examined in 86 patients with probable Alzheimer's disease of minimal to moderate severity. Regional glucose metabolic rates in the posterior cingulate gyri and in the right middle temporal gyrus were significantly correlated with temporal orientation, and the glucose metabolic rate in the right posterior cingulate gyrus was significantly correlated with locational orientation irrespective of age, sex, education, and memory impairment. The results suggest that dysfunction of these structures plays an important part in producing disorientation in patients with Alzheimer's disease.

(F Neurol Neurosurg Psychiatry 1998;64:552-554)
\end{abstract}

Department of Clinical

Neurosciences

N Hirono

E Mori

Y Ikejiri

T Imamura

T Shimomura

M Hashimoto

$\mathrm{H}$ Yamashita

Department of

Neuroimaging

Research, Hyogo

Institute for Aging

Brain and Cognitive

Disorders, Saisho-ko,

Himeji, Japan

K Ishii

M Sasaki

Correspondence to: Dr Nobutsugu Hirono,

Department of Clinical

Neuroscience, Hyogo

Institute for Aging Brain and

Cognitive Disorders, 520

Saisho-ko, Himeji, 670

Japan. Telephone 0081792

8199; email

hirono@hiabcd.gojp

Received 22 May 1997 and in revised form 11

September 1997

Accepted 25 September 1997

Various neurological disorders manifest disorientation for time, place, and person. Orientation may be assessed at the beginning of a mental state examination, although it has rarely been a primary focus of study. Disorientation is commonly regarded as a manifestation of a global brain dysfunction and not as a localisable cognitive function. However, several investigators have reported that disorientation is attributable to specific focal lesions-for example, parietotemporal and thalamic lesions. ${ }^{12}$ Disorientation is a major component of dementia including Alzheimer's disease, and may be extremely severe. The purpose of this study is to determine whether there are any anatomical correlates of disorientation for time and place in patients with Alzheimer's disease by using PET studies of regional cerebral glucose metabolism (CMRglc).

\section{Patients and methods}

According to the following criteria, 86 Japanese patients with Alzheimer's disease were selected from those who were given a short term admission to our hospital for examination between February 1994 and December 1996. After a
95 5511; fax 008179295 complete description of the study was given to the patients and their relatives, written informed consent was obtained. The inclusion criteria included: (1) NINCDS/ADRDA criteria for probable Alzheimer's disease, ${ }^{3}$ and (2) mild to moderate functional severity. The exclusion criteria included: (1) complication of other neurological diseases or physical conditions, (2) any evidence of focal brain lesions on MRI or of cerebral arterial occlusive lesions on MR angiography, (3) presence of severe aphasia, (4) presence of severe cognitive, attentional, or behavioural disorders that would make PET examination difficult (18 candidates were excluded on this basis), and (5) left handedness or equal use of both hands. The mean (SD) age was 70.4 (7.7) years and the mean educational level was 9.2 (2.5) years for 63 women and 23 men. Functional severity as rated by the clinical dementia rating scale $(\mathrm{CDR})^{4}$ was 0.5 (minimal) in 16 patients, 1 (mild) in 52 patients, and 2 (moderate) in 18 patients.

A semiquantitative assessment of orientation orientation subtests of the mini mental state examination (MMSE). ${ }^{5}$ The first comprises five questions regarding time (month, date, year, season, time of day), and the second comprises five questions regarding location (prefecture, city, name of hospital, floor, and number of their room in the hospital). We analysed each measure separately. Each patient's memory function was assessed with the word recall subtest of the Alzheimer's disease assessment scale (ADAS), ${ }^{6}$ in which the retention of a written 10-word list was measured by free immediate recall after each of three learning trials. The recall score was expressed as the mean number of words recalled in three trials. The PET examination was done within one month after the neuropsychological testing.

The CMRglc was measured using PET and ${ }^{18} \mathrm{~F}$-fluorodeoxyglucose as described previously, ${ }^{7}$ with the patient under resting conditions with eyes closed and ears unplugged. Two to four circular regions of interest of $10 \mathrm{~mm}$ diameter were placed on 32 cerebral regions (table). One neuroradiologist blinded to the patients' status made the measurements. To 
Partial correlation coefficients after controlling age, sex, education, and memory function between MMSE subtests of orientation for time and place and regional normalised cerebral glucose metabolism

\begin{tabular}{|c|c|c|c|c|}
\hline \multirow[b]{2}{*}{ Region } & \multicolumn{2}{|c|}{ Orientation for time } & \multicolumn{2}{|c|}{ Orientation for place } \\
\hline & Left side & Right side & Left side & Right side \\
\hline Superior frontal gyrus & 0.088 & 0.046 & 0.059 & 0.131 \\
\hline Middle frontal gyrus & 0.171 & 0.137 & 0.137 & 0.207 \\
\hline Inferior frontal gyrus & 0.200 & 0.158 & 0.188 & 0.163 \\
\hline Basal frontal cortex & 0.142 & 0.198 & 0.112 & 0.188 \\
\hline Anterior cingulate gyrus & 0.131 & 0.085 & 0.067 & 0.206 \\
\hline Posterior cingulate gyrus & $0.384^{\star}$ & $0.381^{\star}$ & 0.291 & $0.368^{\star}$ \\
\hline Superior temporal gyrus & 0.149 & 0.297 & 0.229 & 0.260 \\
\hline Middle temporal gyrus & 0.231 & $0.348^{\star}$ & 0.235 & 0.268 \\
\hline Inferior temporal gyrus & 0.272 & 0.339 & 0.304 & 0.309 \\
\hline Medial temporal region & 0.250 & 0.209 & 0.182 & 0.246 \\
\hline Superior parietal lobule & 0.150 & 0.255 & 0.051 & 0.156 \\
\hline Inferior parietal lobule & 0.229 & 0.333 & 0.149 & 0.218 \\
\hline Lateral occipital cortex & 0.156 & 0.225 & 0.080 & 0.114 \\
\hline Medial occipital cortex & 0.094 & 0.117 & 0.103 & 0.152 \\
\hline Lenticular nucleus & 0.097 & 0.058 & 0.124 & 0.060 \\
\hline Thalamus & 0.072 & 0.039 & 0.065 & 0.080 \\
\hline
\end{tabular}

increase the reliability, the values of CMRglc in each region were averaged. To remove the between patient difference in baseline metabolism, we employed the normalised CMRglc ratio (nCMRglc; CMRglc in a given region: average of CMRglc in the bilateral primary sensorimotor areas).

The relation between disorientation and memory function was explored by multiple linear regression analyses with each MMSE orientation score as a dependent variable, and the ADAS word recall score as an independent variable. Age, sex, and educational attainment were also entered into the model as confounders. To determine whether there were any structural correlates of disorientation, we calculated a Pearson correlation coefficient between nCMRglc of each brain region and each MMSE score. We then evaluated it by using a multiple linear regression partial correlation with each MMSE orientation score as a dependent variable and nCMRglc of each brain region as an independent variable. Demographic variables and memory test performance were incorporated in the model. As 32 repeated comparisons were involved for each subtest, we adopted a conservative $\alpha$ level of $0.05 / 32=0.00156$. All statistical analyses were carried out on SAS release 6.10 (SAS Institute Inc, Cary, NC).

\section{Results}

The mean (SD) MMSE score was 19.9 (4.0). The mean temporal and locational MMSE orientation scores were 2.8 (1.4) and 3.2 (1.2), respectively. The mean ADAS word recall score was 3.8 (1.4). A multiple linear regression analysis showed significant partial correlations between temporal orientation and memory function (partial $r=0.288, \mathrm{p}=0.008$ ), between temporal orientation and age (partial $r$ $=0.235, \mathrm{p}=0.032$ ), and between locational orientation and memory function (partial $r$ $=0.281, \mathrm{p}=0.010$ ).

In the Pearson correlation analyses, the MMSE temporal orientation score correlated significantly with $\mathrm{nCMRglc}$ in the posterior cingulate gyrus $(r=0.400, \mathrm{p}<0.0001)$ and the medial temporal cortex $(r=0.344, \mathrm{p}=0.0012)$
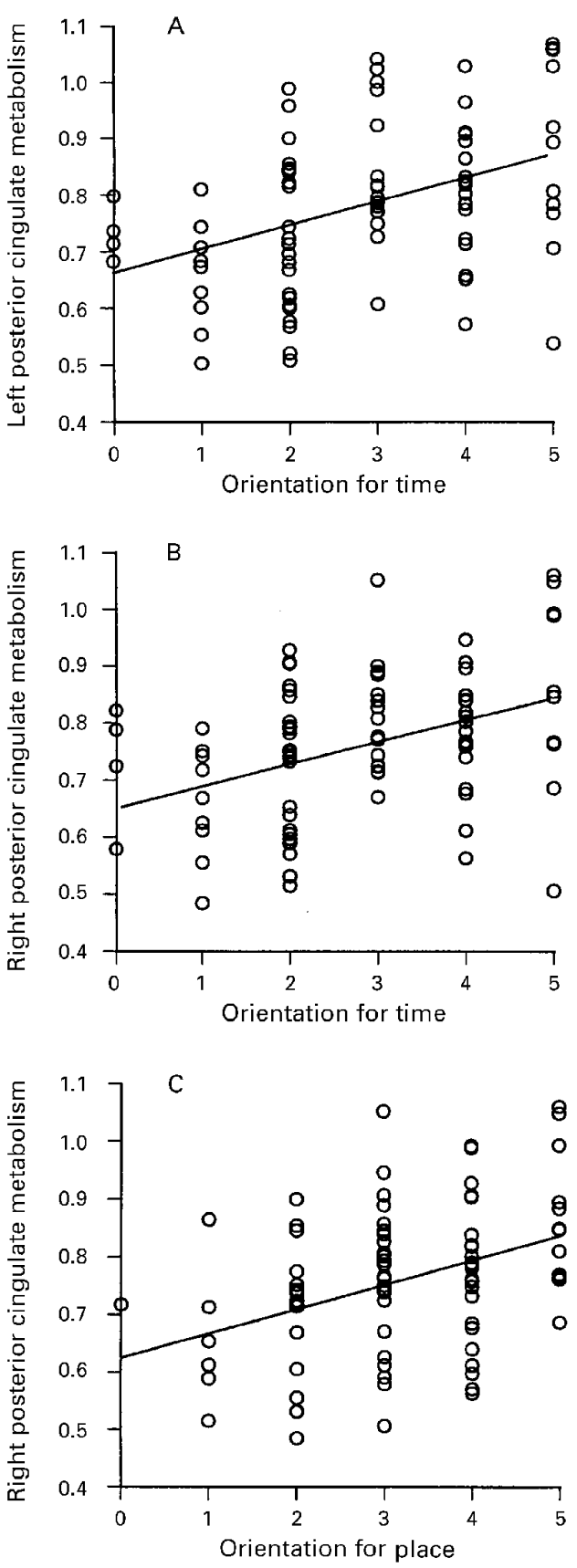

Correlations between $(A)$ temporal orientation score and normalised cerebral glucose metabolism in the posterior cingulate gyrus $(r=0.400, p<0.0001),(B)$ temporal orientation score and right posterior cingulate gyrus metabolism $(r=0.401, p<0.0001)$, and $(C)$ locational orientation score and right posterior cingulate gyrus metabolism $(r=0.385, p=0.0003)$. These relations remained significant after partialling out variance due to age, sex, education, and memory test performance.

in the left side, and the posterior cingulate gyrus $(r=0.401, \mathrm{p}<0.0001)$, middle temporal gyrus $(r=0.388, \mathrm{p}=0.0002)$, inferior temporal gyrus $(r=0.386, \mathrm{p}=0.0002)$, and inferior parietal lobule $(r=0.339, \mathrm{p}=0.0014)$ in the right side, and the MMSE locational orientation score correlated significantly with nCMRglc in the right posterior cingulate gyrus $(r=0.385, \mathrm{p}=$ 0.0003). After controlling for the effects of age, sex, educational attainment, and ADAS word recall score in the multiple regression analyses, 
the temporal orientation score had significant correlations with nCMRglc in the posterior cingulate gyrus in each side and the right middle temporal gyrus, and the locational orientation score had a significant correlation with $\mathrm{nCMRglc}$ in the right posterior cingulate gyrus (table). Scatter plots illustrate the relations between posterior cingulate nCMRglc and orientation (figure).

\section{Discussion}

The present study shows that disorientation for time and place in patients with Alzheimer's disease correlated with memory impairment, as expected, and is likely linked to severity of global disease. Nevertheless, our main finding in this study is that temporal and locational disorientation significantly correlated with the glucose hypometabolism in specific brain regions irrespective of memory impairment. Among these regions, the posterior cingulate gyri were the most relevant sites to temporal and locational orientation.

The roles of the posterior cingulate gyrus in orientation are consistent with a study on multi-infarct dementia by Kitagawa et $a l{ }^{8}$ which showed that disorientation for time and place significantly correlated with regional cerebral blood flow in the right cingulate cortex determined by xenon contrast CT, although they did not specify whether the anterior or posterior cortex was involved. Takahashi et al ${ }^{9}$ also described three patients with selective topographic disorientation caused by focal haemorrhages in the right retrosplenial region. The cingulate gyrus is a principal component of the limbic system. The anterior and posterior cingulate cortices have different cytoarchitectures, and subserve distinctive functions. The anterior cortex primarily subserves executive functions related to the executive attention, ${ }^{10}$ as well as visceromotor and skeletomotor control, and responses to noxious stimuli. ${ }^{11}$ On the other hand, the posterior cortex plays a part in orientation within and interpretation of the environment. ${ }^{11-14}$ There are strong connections between the anterior and posterior cingulate cortices, and therefore, it is likely that the functions of these divisions are coordinated. The posterior cingulate cortex also has dense connections with the medial temporal memory system. These communications likely contribute to the role of the posterior cingulate cortex in orientation. ${ }^{11}$ The posterior cingulate cortex is a major locus that is functionally involved early in the course of Alzheimer's disease. ${ }^{15} 16$ This would account for the severe disorientation for time and place in Alzheimer's disease. The correlations between bilateral posterior cingulate involvement and orientation for time and between right sided posterior cingulate involvement and orientation for place remind us of the different hemispheric specialisations for functions of temporal and locational orientation. Fisher ${ }^{1}$ pointed out that lesions in the non-dominant cerebral hemisphere are associated with locational disorientation, whereas Levin and Benton ${ }^{17}$ pointed out that bilateral lesions are associated with temporal disorientation.

In conclusion, the present study suggests that damage to the posterior cingulate gyri plays an important part in producing disorientation in patients with Alzheimer's disease. This does not necessarily imply that this structure exclusively subserves the function of orientation; orientation is probably a multiple domain function, as disorientation occurs after focal lesions ${ }^{12}$ as well as diffuse encephalopathies. Nevertheless, our results indicate that further studies on the function and pathology of the posterior cingulate gyrus are needed.

1 Fisher CM. Disorientation for place. Arch Neurol 1982;39:33-6.

2 Spiegel EA, Wycis HT, Orchinik CW, et al. The thalamus and temporal orientation. Science 1955;121:771-2.

3 McKhann G, Drachman D, Folstein M, et al. Clinical diagnosis of Alzheimer's disease: report of the N1NCDSADRDA Work Group under the auspices of Department of Health and Human Services Task Force on Alzheimer's Disease. Neurology 1984;34:939-44

4 Hughes CP, Berg L, Danziger WL, et al. A new clinical scale for the staging of dementia. Br $\mathcal{F}$ Psychiatry 1982;140:56672.

5 Folstein MF, Folstein SE, McHugh PR. Mini-mental state: a practical method for grading the cognitive state of patients for the clinician. F Psychiatr Res 1975;12:189-98

6 Mohs RC, Rosen WG, Davis KL. The Alzheimer's disease assessment scale; an instrument for assessing treatment assessment scale; an instrument for assessing

7 Ishii K, Sasaki M, Kitagaki H, et al. Reduction of cerebellar Ishii $\mathrm{K}$, Sasaki $M$, Kitagaki $\mathrm{H}$, et al. Reduction of cerebellar
glucose metabolism in advanced Alzheimer's disease. $\mathcal{f}$ glucose metabolism in ad
Nucl Med 1997;38:925-8

8 Kitagawa Y, Meyer JS, Tachibana H, et al. CT-CBF correlations of cognitive deficits in multi-infarct dementia. Stroke 1984;15:1000-9.

9 Takahashi N, Kawamura M, Shiota J, et al. Pure topographic disorientation due to right retrosplenial lesions. Neurology 1997;49:464-9

10 Posner MI. Attention in cognitive neuroscience: an overview. In: Gazzaniga MS, eds. The cognitive neurosciences. Cambridge: MIT, 1995:615-24

11 Vogt BA, Finch DM, Olson CR. Functional heterogeneity in cingulate cortex: the anterior executive and posterior evaluative regions. Cereb Cortex 1992;2:435-43.

12 Sutherland RJ, Whishaw IQ, Kolb B. Contributions of cingulate cortex to two forms of spatial learning and memory. f Neurosi $1988 ; 8: 1863-72$.

13 Murray EA, Davidson M, Gaffan D, et al. Effects of fornix transection and cingulate cortical ablation on spatial memory in rhesus monkeys. Exp Brain Res 1989;74:173mem.

14 Markowska AL, Olton DS, Murray EA, et al. A comparative analysis of the role of fornix and cingulate cortex in memory: rats. Exp Brain Res 1989;74:187-201.

15 Ishii K, Sasaki M, Yamaji S, et al. Demonstration of decreased posterior cingulate perfusion in mild Alzheimer's disease by means of $\mathrm{H}_{2}^{15} \mathrm{O}$ positron emission tomography. Eur f Nucl Med 1997;24:670-3

16 Minoshima S, Giordani B, Berent S, et al. Metabolic reduction in the posterior cingulate cortex in very early Alzheimer's disease. Ann Neurol 1997;42:85-94.

17 Levin HS, Benton AL. Temporal orientation in patients with brain disease. Appl Neurophysiol 1975;38:56-60. 